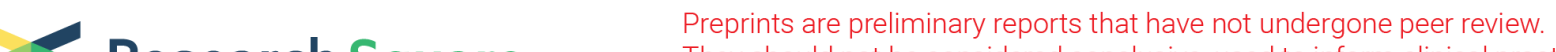 $\begin{array}{ll}\text { Research Square } & \begin{array}{l}\text { They should not be considered conclusive, used to inform clinical practice, } \\ \text { or referenced by the media as validated information. }\end{array}\end{array}$
}

\section{Novel coronavirus mitigation measures implemented by radiotherapy centres in low and middle-income countries: a systematic review}

\section{Andrew Donkor ( $\sim$ Andrew.Donkor@uts.edu.au )}

University of Technology Sydney, Faculty of Health, IMPACCT (Improving Palliative, Aged and Chronic Care through Clinical Research and Translation), NSW Australia https://orcid.org/0000-0002-6073-524X

Vivian Della Atuwo-Ampoh

University of Health and Allied Sciences, School of Allied Health Sciences, Department of Medical Imaging, Ghana

\section{Craig Opie}

Northern Sydney Cancer Centre, Royal North Shore Hospital, Sydney, Australia

\section{Frederick Yakanu}

Korle-Bu Teaching Hospital, National Centre for Radiotheapy and Nuclear Medicine, Accra, Ghana

\section{Dorothy Lombe}

Cancer Diseases Hospital, Zambia

Jamal Khader

King Hussein Cancer Center, Amman, Jordan

\section{Systematic Review}

Keywords: Radiotherapy, Cancer, COVID-19, Implementation, Low and middle-income countries

Posted Date: February 4th, 2021

DOI: https://doi.org/10.21203/rs.3.rs-174503/v1

License: (a) (1) This work is licensed under a Creative Commons Attribution 4.0 International License. Read Full License 


\section{Abstract}

Aim: To identify strategies adopted by radiotherapy centres in low and middle-income countries (LMICs) to mitigate the effects of COVID-19.

Background: Studies summarising COVID-19 mitigation strategies designed and implemented by radiotherapy centres in LMICs to avoid delays, deferrments and interruptions of radiotherapy services are lacking.

Materials and Methods: A systematic review was conducted and reported in accordance with the preferred reporting items for systematic review and meta-analysis guideline. Ovid Embase, Ovid MEDLINE and CINAHL were searched for peer-reviewed articles that reported measures adopted by radiotherapy centres in LMICs to reduce the risk of COVID-19. Information on different strategies were extracted from the included studies and textual narrative synthesis was conducted.

Results: Of the 60 articles retrieved, eleven were included. Majority of the studies were conducted in China. Ten of the included studies employed a qualitative design. Four themes were identified: preparing and equipping staff; reinforcing infection prevention and control policies; strengthening coordination and communication; and maintaining physical distancing. Studies reported that radiotherapy centres have: formed COVID-19 response multidisciplinary team; maximised the use of telehealth; adjusted the layout of waiting areas; divided staff into teams; dedicated a room for isolating suspected cases; and adopted triage systems.

Conclusions: Local adaptation of established global strategies coupled with timely development of guidelines, flexibility and innovation have allowed radiotherapy leaders to continue to deliver radiotherapy services to cancer patients in LMICs during the COVID-19 crisis. Robust and quality data collection must be encouraged in LMICs to provide an evidence-based knowledge for use in the event of another pandemic.

\section{Introduction}

The challenges posed by the 2019 novel coronavirus (COVID-19) are unprecedented, and policy and decision-makers around the globe are dealing with several complexities and uncertainties. No vaccine is currently available. As predicted by mathematical models, the number of severe acute respiratory syndrome coronavirus 2 (SARS-CoV-2) infections increased in the winter season of low and middleincome countries (LMICs) in the southern hemisphere, which is a major concern because of the fragile health systems (Chen et al. 2020; Hopman, Allegranzi \& Mehtar 2020). Governments have demonstrated strong commitments by adopting measures, such as stay-at-home recommendations, business closures and travel restrictions to contain the spread of the virus (Gostin \& Wiley 2020). Some studies have demonstrated that people with cancer are at greater risk from COVID-19 because they often have multiple comorbidities, tend to be older and immunosuppressed due to the disease or its treatment (Saini et al. 2020). In some countries, patients were advised not to visit hospitals because of COVID-19 infection 
risk (Wang \& Zhang 2020). However, this is not a realistic long-term solution for radiotherapy centres and people living with cancer.

Radiotherapy is a critical component of universal health coverage and it is crucial for policy and decisionmakers to prioritise this essential service to avoid disruptive effects from COVID-19. Most radiotherapy centres in LMICs already face resource issues including understaffing and budget constraints. The problems faced in these countries are compounded by misinformation, sociocultural and religious issues, as well as poor sanitary conditions. The delivery of safe and effective radiotherapy services in LMICs in a time of COVID-19 pandemic relies on appropriate policy, healthcare organisation, community and patientlevel interventions. An analysis of an online Twitter discussion by members of the global radiation oncology community highlighted the importance of creating and implementing a programme that prepares, communicates, operates and compensates when radiotherapy is paused during the COVID-19 pandemic (Simcock et al. 2020).

Radiotherapy leaders in high-income coutries (HICs) have generally pursued cautious policies to help staff, cancer patients and their families stay safe at radiotherapy centres during the COVID-19 pandemic (Anderson et al. 2020). Some have adopted: physical distancing by reorganising spaces and reducing number of acompanying family members; triage systems; hand hygiene; use of personal protective equipment (PPE) for all cancer patients regardless of known COVID-19 status; and use of telehealth for training, multidisiciplinary team meetings and follow-ups (Anderson et al. 2020; Belkacemi et al. 2020). Similar strategies are now increasingly being implemented in most LMICs often with limited support, weak infrastructure and less robust local evidence. As a result, the prospect for sustaining and providing equitable radiotherapy service is questionable (Vanderpuye, Elhassan \& Simonds 2020).

To avoid unsustainable service delivery, it is critical that radiotherapy leaders in LMICs formulate and implement COVID-19 policy responses, which align with local priorities, needs and resources. Presently, no studies have been conducted to identify and understand lessons learned based on radiotherapy centres in LMICs' experiences to responding to the COVID-19 pandemic. Therefore, the aim of this study is to identify strategies adopted by radiotherapy centres in LMICs to mitigate the effects of COVID-19.

\section{Materials And Methods}

\section{Materials and Methods}

This systematic review is reported in accordance with the preferred reporting items for systematic review and meta-analysis (PRISMA) guideline (Moher et al. 2009).

\section{Eligibility criteria}

Included studies satisfied three criteria, which were: i) studies had to focus on COVID-19 and radiotherapy services in any country classified as LMIC as defined by the World Bank Group; ii) studies have to 
describe measures adopted to reduce the risk of COVID-19 in a radiotherapy centre; and iii) primary studies published in peer-reviewed journals of any design and in English language. Editorials, opinion pieces, comments, letters, studies focused on high-income settings and studies in languages other than English were excluded.

\section{Information sources}

Three electronic databases were searched, namely: Ovid Embase, Ovid MEDLINE(R) and In-Process \& Other Non-Indexed Citations and CINAHL. A manual search of the reference lists of included studies was performed.

\section{Search strategy}

The search strategy included terms relating to four concepts: i) cancer; ii) radiotherapy; iii) COVID-19; and iv) LMICs. Subject headings, indexed keywords and free text terms relating to the four concepts appearing in titles and/or abstracts were combined using 'AND' or 'OR' (see appendix 1). The initial search strategy was developed in Ovid MEDLINE and adapted for other databases. Databases were searched on 1 July 2020 and updated on 3 August 2020.

\section{Study selection}

Two authors screened titles and abstracts of all citations retrieved for inclusion. Conflicts were resolved through discussion. Studies were excluded if they clearly did not meet the inclusion criteria. Full texts of studies not excluded during the title and abstract screening were obtained.

\section{Data extraction}

An electronic data extraction form was developed, and two reviewers independently abstracted the contents of each included study. The data extracted included: study characteristics; characteristics of strategies implemented; reasons for adopting the strategy; and implementation challenges. Abstracted data were then discussed through an online platform. The risk of bias for each study was appraised using Joanna Briggs Institute Critical Appraisal Checklist for Qualitative Research independently by two reviewers and disagreements were resolved via discussion (Joanna Briggs Institute 2017).

\section{Data synthesis}

Textual narrative synthesis was conducted. Included studies were independently coded by two reviews without a framework. Through thematic analysis, studies were arranged into homogeneous groups, and 
similarities and differences were compared across studies (Lucas et al. 2007). Each study was read and reread to achieve immersion, identify ideas and/or concepts of interest and completed with data reduction and comparison. Emerging themes were explored, refined and any discrepancies were resolved through discussion.

\section{Results}

Of the 60 articles retrieved, seven articles were removed due to duplication. The remaining 53 articles were screened, and 11 articles were excluded based on their title and abstract. Next, 42 articles underwent full-text review and 31 were excluded because they did not satisfy the inclusion criteria, leaving 11 articles for data extraction (see Fig 1). The level of evidence for all of the included studies was low.

\section{Characteristics of included studies}

Characteristics of the included studies are displayed in Table 1. The majority of the studies were conducted in China (Wang et al. 2020; Wei et al. 2020; Wu et al. 2020; Xie et al. 2020). Other studies were conducted in Iran (Aghili, Jafari \& Vand Rajabpoor 2020; Motlagh et al. 2020; Samiee et al. 2020), Brazil (Baldotto et al. 2020b) and Zambia (Lombe et al. 2020). Ten of the included studies employed a qualitative design (Aghili, Jafari \& Vand Rajabpoor 2020; Baldotto et al. 2020b; Gupta et al. 2020; Lombe et al. 2020; Motlagh et al. 2020; Samiee et al. 2020; Wang et al. 2020; Wei et al. 2020; Wu et al. 2020; Xie et al. 2020). A variety of methods were used including case reports (Lombe et al. 2020; Samiee et al. 2020; Wang et al. 2020; Wei et al. 2020; Xie et al. 2020), experts consensus (Aghili, Jafari \& Vand Rajabpoor 2020; Baldotto et al. 2020b; Gupta et al. 2020; Motlagh et al. 2020), cross-sectional survey (Saab et al. 2020) and critical review (Wu et al. 2020). There is a strong support for hypofractionated radiotherapy. Four studies reported the radiotherapy fractionation schedules implemented during the COVID-19 pandemic period (see Table 2) (Aghili, Jafari \& Vand Rajabpoor 2020; Baldotto et al. 2020a; Gupta et al. 2020; Lombe et al. 2020).

\section{Thematic analysis}

Four themes emerged: preparing and equipping staff; reinforcing infection prevention and control policies; strengthening coordination and communication; and maintaining physical distancing. Different mitigation measures have also been summarised in a matrix with 21 subthemes (see Fig 2).

\section{Preparing and equipping staff}

Within this theme, studies acknowledged the importance of preparing and equipping staff and supporting staff to manage emerging psychosocial risks. Five studies reported providing staff with initial and ongoing education and training on correct use, maintenance and disposal of PPE, hand hygiene, 
disinfection procedures, isolation policies, symptoms of COVID-19 and latest national guidelines on diagnosing and managing COVID-19 (Gupta et al. 2020; Lombe et al. 2020; Motlagh et al. 2020; Wang et al. 2020; Wei et al. 2020; Wu et al. 2020). Strategies frequently mentioned for delivering training included educational pamphlets and a blend of web-based learning and hands-on experience (Gupta et al. 2020; Lombe et al. 2020; Motlagh et al. 2020; Wei et al. 2020). In the early phase of the pandemic, lack of effective infection control programmes and weak enforcement mechanisms were major factors associated with increased person-to-person transmission resulting in several radiation oncologists, two radiation therapists and an unknown number of patients been infected with COVID-19 (Wu et al. 2020).

Eight studies reported that preparing and equipping staff with programmes, policies and resources, such as non-contact temperature assessment devices, telehealth equipment, PPE, sterilisation technologies and disinfectants were crucial to support and protect staff to deliver safe radiotherapy (Aghili, Jafari \& Vand Rajabpoor 2020; Gupta et al. 2020; Lombe et al. 2020; Samiee et al. 2020; Wang et al. 2020; Wei et al. 2020; Wu et al. 2020; Xie et al. 2020). Lack of access to affordable PPE and stock shortages were obstacles to cancer care delivery (Saab et al. 2020). The recommended solution included improving communication lines for better centralised ordering and distribution of PPE (Wei et al. 2020). Another study indicated that staff managing their mental health and psychosocial well-being during the COVID-19 crisis was essential due to increased stress (Saab et al. 2020). However, the findings also showed that helpful coping strategies are significantly needed for staff, cancer patients and their families.

\section{Reinforcing infection prevention and control policies}

All studies reported radiotherapy management commitment to reinforcing infection prevention and control policies to reduce the risk of transmission of COVID-19 at radiotherapy centres (Aghili, Jafari \& Vand Rajabpoor 2020; Baldotto et al. 2020b; Gupta et al. 2020; Lombe et al. 2020; Motlagh et al. 2020; Saab et al. 2020; Samiee et al. 2020; Wang et al. 2020; Wei et al. 2020; Wu et al. 2020; Xie et al. 2020). In most of these studies, staff wearing proper PPE (e.g. gowns, masks, face shields and gloves) (Gupta et al. 2020; Lombe et al. 2020; Motlagh et al. 2020; Wang et al. 2020; Wei et al. 2020; Wu et al. 2020; Xie et al. 2020), keeping treatment and other rooms well-ventilated (Wang et al. 2020; Wei et al. 2020; Xie et al. 2020), disinfecting surfaces that patients and staff are in constant contact with, such as treatment couch (Motlagh et al. 2020; Samiee et al. 2020; Wang et al. 2020; Wei et al. 2020; Wu et al. 2020; Xie et al. 2020), adhering to strict hand hygiene (Gupta et al. 2020; Lombe et al. 2020; Motlagh et al. 2020; Samiee et al. 2020; Wang et al. 2020; Wei et al. 2020; Xie et al. 2020) and managing waste effectively (Wang et al. 2020; Wei et al. 2020; Xie et al. 2020) were approaches frequently reported for creating safe infection control practice. Four studies reported cancer patients and their accompanying carers were required to wear face masks when entering the radiotherapy facility to protect themselves in a preventive manner (Gupta et al. 2020; Motlagh et al. 2020; Samiee et al. 2020; Wei et al. 2020). Shortages of face masks in the community was a critical barrier. As a result, one study recommended providing cancer patients with sanitary packages containing face masks, gloves and hand sanitisers on arrival (Motlagh et al. 2020). 
All studies highlighted the importance of adopting triage systems to sort, assess and prioritise staff, cancer patients and their carers entering the radiotherapy centre (Aghili, Jafari \& Vand Rajabpoor 2020; Baldotto et al. 2020b; Gupta et al. 2020; Lombe et al. 2020; Motlagh et al. 2020; Saab et al. 2020; Samiee et al. 2020; Wang et al. 2020; Wei et al. 2020; Wu et al. 2020; Xie et al. 2020). From these studies designing a flow chart helped facilitate the COVID-19 screening and testing process (Samiee et al. 2020; Xie et al. 2020). Daily body temperature check at the entrance and asking cancer patients and their carers series of questions were critical to identifying if they present symptoms indicative of COVID-19. Moreover, further studies showed that most radiotherapy centres in LMICs had implemented suspect case testing for staff, cancer patients and carers who have had close contact with a COVID-19 case and/or have symptoms of COVID-19 (Aghili, Jafari \& Vand Rajabpoor 2020; Gupta et al. 2020; Motlagh et al. 2020; Saab et al. 2020; Samiee et al. 2020; Wang et al. 2020; Wei et al. 2020; Xie et al. 2020). Overall, a suspect case testing included chest computed tomography (CT) scan and polymerase chain reaction (PCR) test (Aghili, Jafari \& Vand Rajabpoor 2020; Saab et al. 2020; Xie et al. 2020). Often studies highlighted that if results were abnormal, suspected cases were immediately transported to a designated infectious disease institution equipped to manage suspected or confirmed case of COVID-19 in line with national guidelines (Wang et al. 2020; Xie et al. 2020).

Four studies reported that some radiotherapy centres strategy to mitigate the spread of the COVID-19 includes dividing the facility into three zones: clean zone; semi-contaminated zone; and contaminated zone (Motlagh et al. 2020; Wang et al. 2020; Wei et al. 2020; Wu et al. 2020). Administrative offices, medical physics and dosimetry offices were reported as clean zones while semi-contaminated zones included changing and restrooms, patient corridors and waiting areas. Contaminated zones comprised treatment vaults, simulation rooms, consulting rooms, console areas and front desk areas (Wei et al. 2020). Posting reminder signs for staff to remove contaminated PPE before leaving designated contaminated zones was essential to avoid cross-contamination (Samiee et al. 2020; Wei et al. 2020).

\section{Strengthening coordination and communication}

Studies recognised that strengthening good communications among staff, and between staff and cancer patients can enhance coordination and mobilise support around policy to provide safe radiotherapy services, including brachytherapy for cancer patients with the maximum level of protection (Aghili, Jafari \& Vand Rajabpoor 2020). A belief commonly reported was the perception that eliminating COVID-19 soon was a false hope. Therefore, most radiotherapy centres have undertaken efforts that include forming COVID-19 response multidisciplinary team intended to improve internal coordination and communication by rapidly developing clinical guidelines (e.g. lung, breast, cervical and prostate cancers), policies and channel advice on health and safety during the COVID-19 pandemic (Lombe et al. 2020; Wei et al. 2020). Some studies revealed that the COVID-19 response multidisciplinary team was responsible for coordinating all aspects of infection control activities, which included staff training, radiotherapy workflow modification and management of PPE (Lombe et al. 2020; Wei et al. 2020). 
Further initiatives to strengthening coordination and communication included establishing a hotline for cancer patients and their families as well as using social media (e.g. WhatsApp) for cancer patients to notify the designated staff member responsible for handling complaints (Samiee et al. 2020). Other studies reported discussing radiotherapy with new cancer patients via telehealth (either phone or video) and making decisions on avoiding, deferring or shortening treatment (Samiee et al. 2020). All studies acknowledged that COVID-19 is a challenging situation and information keeps changing as new guidelines are developed. Therefore, providing weekly information sessions for all staff to update and advice staff on the latest changes to cancer and COVID-19 guidelines was essential (Motlagh et al. 2020).

\section{Maintaining physical distancing}

All studies reported that radiotherapy centres had implemented multiple strategies to allow for physical distancing to reduce the likelihood of exposure to micro-droplets infections (Gupta et al. 2020; Lombe et al. 2020; Motlagh et al. 2020; Saab et al. 2020; Samiee et al. 2020; Wang et al. 2020; Wei et al. 2020; Xie et al. 2020). Adjusting the layout of waiting areas, rethinking appointments to reduce the capacity of patient flow (e.g. two patients for every 30-40 minutes timeslot), allowing a single patient in a consultation room at a time, designating separate entrance and exit and restricting the number of carers accompanying patients were among most frequently mentioned strategies to create space while maintaining a distance of at least one meter around individuals (Lombe et al. 2020; Motlagh et al. 2020; Samiee et al. 2020; Wang et al. 2020; Xie et al. 2020).

Four studies reported that splitting staff into two or three separate teams helped to reduce the number of staff on-site at any given time (Gupta et al. 2020; Wang et al. 2020; Wei et al. 2020; Xie et al. 2020). Particularly, a single study indicated that schedules were modified to ensure there was no overlap of staff (Wei et al. 2020). Similarly, some radiotherapy centres have developed a flexible arrangement that allows certain staff, such as medical physicists and dosimetrists to work from home. Staff with proper equipment and training were able to perform remote treatment planning (Wei et al. 2020). In one study, simulation sessions were reduced to two days per week (Samiee et al. 2020).

Five studies reported that radiotherapy centres had arranged a dedicated space for isolation of any patient, carer and/or staff who develops COVID-19 symptoms at the radiotherapy site (Motlagh et al. 2020; Samiee et al. 2020; Wang et al. 2020; Wei et al. 2020; Xie et al. 2020). The availability of isolation room helped kept suspected cases separate while awaiting transfer to a dedicated COVID-19 facility. Some studies showed that staff and other patients who may have been in contact with confirmed COVID19 case were asked to practice self-quarantine (Wang et al. 2020).

A large number of studies indicated increased use of telehealth instead of face-to-face consultations to mitigate the spread of the COVID-19 (Baldotto et al. 2020b; Gupta et al. 2020; Lombe et al. 2020; Motlagh et al. 2020; Saab et al. 2020; Samiee et al. 2020; Wang et al. 2020). Telehealth provided a crucial advantage in the delivery of multidisciplinary team meetings, follow-ups with patients after treatment 
irrespective of location, triage for patient booking appointment and education (Baldotto et al. 2020b; Gupta et al. 2020; Lombe et al. 2020; Motlagh et al. 2020; Saab et al. 2020; Samiee et al. 2020; Wang et al. 2020). Inherently, issues relating to implementing and sustaining telehealth in radiotherapy, as well as infrastructure that facilitate equitable telehealth access during COVID-19 were not examined in the included studies. Without sustainability planning and actions to address barriers to accessing telehealth, radiotherapy leaders risk creating telehealth programmes that exclude specific individuals and populations, such as rural patients.

\section{Discussion}

This systematic review was conducted to synthesise the current evidence on measures implemented by radiotherapy centres in LMICs to reduce the risk of contracting COVID-19. The literature indicates that radiotherapy centres in LMICs have implemented multi-component strategies to: reinforce infection prevention and control policies; prepare and equip staff; strengthen coordination and communication; and maintain physical distancing. The most commonly reported strategies included: using telehealth; wearing PPE; hand hygiene, splitting staff into separate teams; zoning; working from home; training staff; weekly information sessions; developing guidelines, procedures and protocols; adjusting the layout of waiting areas; designating separate entrance and exit; restricting the number of accompanying carers; adopting triage systems; COVID-19 testing; and arranging a dedicated space for isolation. However, it is difficult to determine the effectiveness of these strategies because included studies have methodological limitations and provided minimal information about their impact.

Radiotherapy staff are committed to providing safe services to cancer patients and must be protected against COVID-19 at all times (Belkacemi et al. 2020). There is evidence showing that more than 3,300 healthcare workers in China have been infected as of March 2020 (Lancet 2020). Recognising that transmission occurs via symptomatic and asymptomatic individuals, preparation and protection are especially important. Effective protection of radiotherapy staff is likely to be dependent on a combination of special training on infection control, availability and proper use of PPE, provision of safe air (good ventilation) and administrative controls, such triage systems to identify, isolate, investigate and effectively manage individuals who may present COVID-19 symptoms. Developing and implementing evidence-based COVID-19 control policies and procedures provide guidance for reducing the risk of transmission of COVID-19 in the radiotherapy setting (Simcock et al. 2020). To protect staff, cancer patients and their families, radiotherapy leaders have both administrative and supervisory responsibilities to periodically evaluate and revise COVID-19 infection control policies and procedures. The review shows that a radiotherapy centre COVID-19 infection control policy ideally needs to update cleaning and disinfection procedures and outline arrangements for isolating and transferring suspected cases to a dedicated COVID-19 facility for further investigation and management, so patients with COVID-19 do not infect others.

In many LMICs, cancer patients and their accompanying carers are required to wear facemasks when entering a radiotherapy centre to provide additional protection when needed. However, it presents 
additional communication challenges for both staff and cancer patients. Radiotherapy leaders taking advantage of technology, such as speech applications on smartphones can help address communication challenges. As COVID-19 spreads globally, demand for PPE has increased creating shortage problems. It is estimated that the need for surgical masks, gloves and face shields could reach 2.2 billion, 1.1 billion and 8.8 million respectively through the end of 2020 (UNICEF 2020). Other studies have shown that adequate production and distribution of PPE are crucial to caring for patients during COVID-19 pandemic (Ranney, Griffeth \& Jha 2020; Rowan \& Laffey 2020). Our review supports those findings. For example, to optimise the supply of PPE, radiotherapy centres in LMICs could implement strategies such as: creating partnerships with not-for-profit organisations and/or national coalition of centres in cancer care ; training staff on PPE donning and doffing procedures; storing PPE in secured and monitored locations; providing facemasks to patients at the entrance; ensuring extended use of facemasks; and limiting face-to-face contact by maximising the use of telehealth.

Findings from the review suggest that the emergence of COVID-19 has caused a rapid adoption of telehealth in radiotherapy services, with no information on sustainability. This finding is consistent with previous studies, which recommend careful planning, implementation and evaluation to ensure equity and sustainability of telehealth after the COVID-19 crisis (Fryer et al. 2020; Monaghesh \& Hajizadeh 2020). Telehealth in radiotherapy services helps maintain physical distancing. New cancer patient consultations, multidisciplinary team meetings and follow-up are made possible through telehealth. If telehealth is to have significant impact in radiotherapy services in LMICs, leaders need to: develop education and training for cancer patients to acquire the requisite digital skills; advocate for free or less expensive internet service charges; secure funding to expand and sustain telehealth; and identify cancer patients who are unable to engage in video follow-up due to lack of device (Nouri et al. 2020).

It is worth noting that COVID-19 practical radiotherapy treatment recommendations for cancers, such as breast, cervix, lung, head and neck, prostate and colorectal have been published by various international and national organisations and societies (Aghili et al. 2020; Guckenberger et al. 2020; Thomson et al. 2020). For palliative non small cell lung carcinoma, 8-10 Gy/1 fraction is strongly recommended (Portaluri et al. 2020). Consensus-based recommendations from American Society for Radiation Oncology and European Society for Radiology and Oncology strongly suggests $50 \mathrm{~Gy} / 16$ fractions for early larynx cancer (T1N0) (Thomson et al. 2020). However, more robust evidence is required to support some of the fractionations for head and neck tumors (Portaluri et al. 2020).

\section{Strengths And Limitations}

This is the first systematic review to synthesise the evidence on COVID-19 mitigation measures implemented by radiotherapy centres in LMICs. Multiple electronic databases were searched to minimise the risk of missing studies. However, it is possible that other studies were not identified because of the rapidly changing field and publication rate. Majority of the studies were from China and it may not be possible to generalise the results. None of the studies described an intervention that uses incentive to both encourage self-isolation and prepare staff to face a health crisis, such as COVID-19. 


\section{Conclusion}

As radiotherapy centres in LMICs navigate through and beyond COVID-19, it is important to seize the opportunity to recognise and address challenges to strengthen radiotherapy workforce. Engaging and equipping staff, cancer patients and their families with the necessary resources, information, knowledge and skills are critical success factors to ensure adherence to measures, such as physical distancing and quarantine. A coordinated approach to communicating messages to staff, cancer patients and their families is equally important to prevent confusion, mistrust and uncertainty. Subsequent studies are needed to explore and explain the effectiveness of the strategies identified in the review. The real strength of radiotherapy leaders to reduce the risk of COVID-19 in radiotherapy centres in LMICs comes from their ability to be flexible and innovative. New models for radiotherapy services may emerge from the COVID19 pandemic. Robust and high quality data collection must be encouraged in LMICs to provide an evidence-based knowledge for use in the event of another pandemic.

\section{Abbreviations}

LMICs: low and middle-income countries; COVID-19: coronavirus disease 2019; PRISMA: preferred reporting items for systematic reviews and meta-analysis; PPE: personal protective equipment; Fx: fraction; Gy: Gray

\section{Declarations}

\section{Ethics approval and consent to participate}

This article is based on a secondary analysis of the existing literature and does not contain any studies with human participants or animals performed by any of the authors. The PRISMA guideline for conducting systematic and meta-analysis was followed.

\section{Consent for publication}

Not applicable.

\section{Competing interests}

The authors declare that they have no competing interest.

\section{Authors contributions}

All authors contributed to the study design, manuscript development, editing and completion of the manuscript. The article search and management were performed by AD. Article screening and data 
extraction were completed by AD and VDA-A. Data synthesis was performed by AD and VDA-A and consensus discussion with the team. All the authors read and approved the final manuscript.

\section{References}

Aghili, M., Ghalehtaki, R., Mousavi Darzikolaee, N., Jafari, F. \& Moshtaghian, M. 2020, 'Radiotherapy and COVID-19: Practical recommendations from Iran', Radiotherapy and Oncology, vol. 149, pp. 70-1.

Aghili, M., Jafari, F. \& Vand Rajabpoor, M. 2020, 'Brachytherapy during the coronavirus disease 2019 Lessons from Iran', Brachytherapy., pp. e412-e4.

Anderson, N., Thompson, K., Andrews, J., Chesson, B., Cray, A., Phillips, D., Ryan, M., Soteriou, S., Trainor, G. \& Touma, N. 2020, 'Planning for a pandemic: Mitigating risk to radiation therapy service delivery in the COVID-19 era', Journal of Medical Radiation Sciences, pp. 1-6.

Baldotto, C., Gelatti, A., Accioly, A., Mathias, C., Mascarenhas, E., Carvalho, H., Faroni, L., Araujo, L.H., Zukin, M., Gadia, R., Terra, R.M., Haddad, R., Lima, V.C. \& Castro-Junior, G. 2020a, 'Lung Cancer and the COVID-19 pandemic: Recommendations from the Brazilian Thoracic Oncology Group', Clinics (Sao Paulo, Brazil), vol. 75, p. e2060.

Baldotto, C., Gelatti, A., Accioly, A., Mathias, C., Mascarenhas, E., Carvalho, H., Faroni, L., Araujo, L.H., Zukin, M., Gadia, R., Terra, R.M., Haddad, R., Lima, V.C. \& Castro-Junior, G. 2020b, 'Lung Cancer and the COVID-19 pandemic: recommendations from the Brazilian Thoracic Oncology Group', Clinics, vol. 75, pp. e2060-e5.

Belkacemi, Y., Grellier, N., Ghith, S., Debbi, K., Coraggio, G., Bounedjar, A., Samlali, R., Tsoutsou, P.G., Ozsahin, M., Chauvet, M.P., Turkan, S., Boussen, H., Kuten, A., Tesanovic, D., Errihani, H., Benna, F., Bouzid, K., Idbaih, A., Mokhtari, K., Popovic, L., Spano, J.P., Lotz, J.P., Cherif, A., To, H., Kovcin, V., Arsovski, O., Beslija, S., Dzodic, R., Markovic, I., Vasovic, S., Stamatovic, L., Radosavljevic, D., Radulovic, S., Vrbanec, D., Sahraoui, S., Vasev, N., Stojkovski, I., Risteski, M., Freixa, S.V., Krengli, M., Radosevic, N., Mustacchi, G., Filipovic, M., Kerrou, K., Taghian, A.G., Todorovic, V., Geara, F. \& Gligorov, J. 2020, 'A review of the international early recommendations for departments organization and cancer management priorities during the global COVID-19 pandemic: applicability in low- and middle-income countries', European Journal of Cancer, vol. 135, pp. 130-46.

Chen, T.-M., Rui, J., Wang, Q.-P., Zhao, Z.-Y., Cui, J.-A. \& Yin, L. 2020, 'A mathematical model for simulating the phase-based transmissibility of a novel coronavirus', Infectious Diseases of Poverty, vol. 9, no. 1, pp. 24-31.

Fryer, K., Delgado, A., Foti, T., Reid, C.N. \& Marshall, J. 2020, 'Implementation of obstetric telehealth during COVID-19 and beyond', Maternal and Child Health Journal, pp. e1-e7. 
Gostin, L.O. \& Wiley, L.F. 2020, 'Governmental public health powers during the COVID-19 pandemic: stayat-home orders, business closures, and travel restrictions', JAMA, pp. e1-e2.

Guckenberger, M., Belka, C., Bezjak, A., Bradley, J., Daly, M.E., DeRuysscher, D., Dziadziuszko, R., FaivreFinn, C., Flentje, M., Gore, E., Higgins, K.A., Iyengar, P., Kavanagh, B.D., Kumar, S., Le Pechoux, C., Lievens, Y., Lindberg, K., McDonald, F., Ramella, S., Rengan, R., Ricardi, U., Rimner, A., Rodrigues, G.B., Schild, S.E., Senan, S., Simone li, C.B., Slotman, B.J., Stuschke, M., Videtic, G., Widder, J., Yom, S.S. \& Palma, D. 2020, 'Practice Recommendations for Lung Cancer Radiotherapy During the COVID-19 Pandemic: An ESTROASTRO Consensus Statement', International Journal of Radiation Oncology, Biology, Physics, vol. 107, no. 4, pp. 631-40.

Gupta, M., Ahuja, R., Gupta, S., Joseph, D., Pasricha, R., Verma, S. \& Pandey, L. 2020, 'Running of high patient volume radiation oncology department during covid-19 crisis in India: Our institutional strategy', Radiation Oncology Journal, vol. 38, no. 2, pp. 93-8.

Hopman, J., Allegranzi, B. \& Mehtar, S. 2020, 'Managing COVID-19 in low- and middle-income countries', JAMA, vol. 323 , no. 16 , pp. 1549-50.

Joanna Briggs Institute 2017, The Joanna Briggs Institute Critical Appraisal tools for use in JBI systematic reviews checklist for qualitative research, <https://joannabriggs.org/sites/default/files/201905/JBI_Critical_Appraisal-Checklist_for_Qualitative_Research2017_0.pdf>.

Lancet 2020, 'COVID-19: protecting health-care workers', Lancet (London, England), vol. 395, no. 10228, p. 922.

Lombe, D.C., Mwaba, C.K., Msadabwe, S.C., Banda, L., Mwale, M., Pupwe, G., Kamfwa, P., Kanduza, M., Munkupa, H., Maliti, B., Simbeye, K., Hachizo, P., Lin, L., Chiao, E. \& Lishimpi, K. 2020, 'Zambia's National Cancer Centre response to the COVID-19 pandemic-an opportunity for improved care', ecancermedicalscience, vol. 14, pp. 1051-8.

Lucas, P.J., Baird, J., Arai, L., Law, C. \& Roberts, H.M. 2007, 'Worked examples of alternative methods for the synthesis of qualitative and quantitative research in systematic reviews', BMC Medical Research Methodology, vol. 7, no. 1, pp. 4-9.

Moher, D., Liberati, A., Tetzlaff, J., Altman, D.G. \& Prisma Group 2009, 'Preferred reporting items for systematic reviews and meta-analyses: the PRISMA statement', PLoS Medicine, vol. 6, no. 7, pp. e1000097-e103.

Monaghesh, E. \& Hajizadeh, A. 2020, 'The role of telehealth during COVID-19 outbreak:: a systematic review based on current evidence', Research Square, pp. 1-20.

Motlagh, A., Yamrali, M., Azghandi, S., Azadeh, P., Vaezi, M., Ashrafi, F., Zendehdel, K., Mirzaei, H., Basi, A., Rakhsha, A., Seifi, S., Tabatabaeefar, M., Elahi, A., Pirjani, P., Shoar, L.M., Nadarkhani, F., Khoshabi, M., 
Bahar, M., Esfahani, F. \& Fudazi, H. 2020, 'COVID19 prevention and care: a cancer specific guideline', Archives of Iranian Medicine, vol. 23, no. 4, pp. 255-64.

Nouri, S., Khoong, E.C., Lyles, C.R. \& Karliner, L. 2020, 'Addressing equity in telemedicine for chronic disease management during the Covid-19 pandemic', NEJM Catalyst Innovations in Care Delivery, vol. 1, no. 3, pp. 1-13.

Portaluri, M., Barba, M.C., Musio, D., Tramacere, F., Pati, F. \& Bambace, S. 2020, 'Hypofractionation in COVID-19 radiotherapy: A mix of evidence based medicine and of opportunities', Radiotherapy and Oncology, vol. 150, pp. 191-4.

Ranney, M.L., Griffeth, V. \& Jha, A.K. 2020, 'Critical supply shortages-the need for ventilators and personal protective equipment during the Covid-19 pandemic', New England Journal of Medicine, vol. 382, no. 18 , pp. e41-e3.

Rowan, N.J. \& Laffey, J.G. 2020, 'Challenges and solutions for addressing critical shortage of supply chain for personal and protective equipment (PPE) arising from Coronavirus disease (COVID19) pandemic - case study from the Republic of Ireland', Science of The Total Environment, vol. 725, pp. 138532-40.

Saab, R., Obeid, A., Gachi, F., Boudiaf, H., Sargsyan, L., Al-Saad, K., Javakhadze, T., Mehrvar, A., Abbas, S.S., Abed Al-Agele, Y.S., Al-Haddad, S., Al Ani, M.H., Al-Sweedan, S., Al Kofide, A., Jastaniah, W., Khalifa, N., Bechara, E., Baassiri, M., Noun, P., El-Houdzi, J., Khattab, M., Sagar Sharma, K., Wali, Y., Mushtaq, N., Batool, A., Faizan, M., Raza, M.R., Najajreh, M., Mohammed Abdallah, M.A., Sousan, G., Ghanem, K.M., Kocak, U., Kutluk, T., Demir, H.A., Hodeish, H., Muwakkit, S., Belgaumi, A., Al-Rawas, A.H. \& Jeha, S. 2020, 'Impact of the coronavirus disease 2019 (COVID-19) pandemic on pediatric oncology care in the Middle East, North Africa, and West Asia Region: A report from the Pediatric Oncology East and Mediterranean (POEM) Group', Cancer., pp. 1-11.

Saini, K.S., de las Heras, B., de Castro, J., Venkitaraman, R., Poelman, M., Srinivasan, G., Saini, M.L., Verma, S., Leone, M. \& Aftimos, P. 2020, 'Effect of the COVID-19 pandemic on cancer treatment and research', The Lancet Haematology, vol. S2352-3026, no. 20, pp. 30123-5.

Samiee, S., Hadjilooei, F., Alamolhoda, M. \& Akhlaghpoor, S. 2020, 'New policy and regulations for a radiology-oncology center during the COVID-19 outbreak in Tehran, Iran', Advances in Radiation Oncology., pp. 1-8.

Simcock, R., Thomas, T.V., Mercy, C.E., Filippi, A.R., Katz, M.A., Pereira, I.J. \& Saeed, H. 2020, 'COVID-19: global radiation oncology's targeted response for pandemic preparedness', Clinical and Translational Radiation Oncology, pp. 55-68.

Thomson, D.J., Palma, D., Guckenberger, M., Balermpas, P., Beitler, J.J., Blanchard, P., Brizel, D., Budach, W., Caudell, J., Corry, J., Corvo, R., Evans, M., Garden, A.S., Giralt, J., Gregoire, V., Harari, P.M., Harrington, K., 
Hitchcock, Y.J., Johansen, J., Kaanders, J., Koyfman, S., Langendijk, J.A., Le, Q.-T., Lee, N., Margalit, D., Mierzwa, M., Porceddu, S., Soong, Y.L., Sun, Y., Thariat, J., Waldron, J. \& Yom, S.S. 2020, 'Practice Recommendations for Risk-Adapted Head and Neck Cancer Radiation Therapy During the COVID-19 Pandemic: An ASTRO-ESTRO Consensus Statement', International Journal of Radiation Oncology*Biology*Physics, vol. 107, no. 4, pp. 618-27.

UNICEF 2020, COVID-19 impact assessment and outlook on personal protective equipment viewed 30/7/2020, <https://www.unicef.org/supply/stories/covid-19-impact-assessment-and-outlook-personalprotective-equipment>.

Vanderpuye, V., Elhassan, M.M.A. \& Simonds, H. 2020, 'Preparedness for COVID-19 in the oncology community in Africa', The Lancet Oncology, vol. 21, no. 5, pp. 621-2.

Wang, H. \& Zhang, L. 2020, 'Risk of COVID-19 for patients with cancer', The Lancet Oncology, vol. S14702045, no. 21, p. e181.

Wang, W., Liu, X., Zhang, F., Qiu, J. \& Hu, K.E. 2020, 'Radiation therapy during the COVID-19 pandemic: experience from Beijing, China', In Vivo, vol. 34, no. 3 Suppl, pp. 1675-80.

Wei, W., Zheng, D., Lei, Y., Wu, S., Verma, V., Liu, Y., Wei, X., Bi, J., Hu, D. \& Han, G. 2020, 'Radiotherapy workflow and protection procedures during the Coronavirus Disease 2019 (COVID-19) outbreak: Experience of the Hubei Cancer Hospital in Wuhan, China', Radiotherapy and Oncology, vol. 148, pp. 20310.

Wu, S., Zheng, D., Liu, Y., Hu, D., Wei, W. \& Han, G. 2020, 'Radiation therapy care during a major outbreak of COVID-19 in Wuhan', Advances in Radiation Oncology, pp. 1-3.

Xie, C., Wang, X., Liu, H., Bao, Z., Yu, J., Zhong, Y. \& Chua, M.L.K. 2020, 'Infection control of 2019 novel corona virus disease (COVID-19) in cancer patients undergoing radiotherapy in Wuhan', Available at SSRN 3556646, pp. 1-22.

\section{Tables}

Table 1: Summary of studies included in the review 


\begin{tabular}{|c|c|c|c|}
\hline Authors & Aim & Design & Measures \\
\hline $\begin{array}{l}\text { Aghili, } \\
\text { Jafari \& } \\
\text { Vand } \\
\text { Rajabpoor } \\
2020\end{array}$ & $\begin{array}{l}\text { To consider some of the best feasible brachytherapy } \\
\text { regimes during the pandemic }\end{array}$ & $\begin{array}{c}\text { Expert } \\
\text { consensus }\end{array}$ & $\begin{array}{l}\text { Iran } \\
\text { - Adoption of a triage } \\
\text { system } \\
\text { - COVID-19 testing } \\
\text { - Interruption of the } \\
\text { treatment for at least two } \\
\text { weeks for confirmed } \\
\text { COVID-19 cases } \\
\text { - Escalation of dose per } \\
\text { fraction and reducing the } \\
\text { number of fractions }\end{array}$ \\
\hline
\end{tabular}

Baldotto To outline guidelines that are both consensus-based,

et al. representing major cancer treatment medical societies,

2020 and localised, taking into account local needs for

managing these patients.
Gupta et To clarify the common doubts being faced by the high-

al. 2020

volume centres regarding the functioning of the department, the treatment of patients and the safety of radiation personnel

Expert
consensus

consensus

\section{Brazil}

- Discuss stereotactic ablative radiotherapy (SABR) in patients with stage I and II disease, especially if $\geq 70$ years of age and at higher surgical risk

- Prefer hypofractionated radiotherapy when possible

- Use of PPE

- Adoption of a triage system

- COVID-19 testing

- Use of telehealth for multidisciplinary team meetings

$\begin{array}{cc}\text { Expert } & \begin{array}{c}\text { India } \\ \text { consensus }\end{array} \\ & \text { Clean waiting area with } \\ & \text { adequate distancing } \\ & \text { between the waiting } \\ & \text { benches and the patients } \\ \bullet & \text { Time slots defined for } \\ & \text { patients and treating no } \\ & \text { more than } 5 \text { patients per } \\ & \text { hour } \\ & \text { Stringent use mask and } \\ \text { follow standard hand } \\ \text { hygiene }\end{array}$


- Education for staff on the correct use of PPE

- Thermal screening of all patients/staff at radiation premises

- Staff divided into two groups

- Use of telemedicine to minimise patients' visits

- The potential benefits and risks of altered fractionations discussed with patients

- Minimal use of radiation accessories

- Withholding postgraduate teaching/online teaching platform

- COVID-19 testing for patients with travel history

- Home quarantine for staff with direct contact with confirmed COVID-19 case

\begin{tabular}{ll}
\hline Lombe et & To report the response of a comprehensive cancer centre \\
al 2020 & in a lower-middle income country to prevent COVID-19 \\
& transmission and how the implementation of pragmatic \\
& strategies have served as a springboard to improve \\
& cancer services beyond the COVID-19 pandemic.
\end{tabular}

Lombe et To report the response of a comprehensive cancer centre in a lower-middle income country to prevent COVID-19 strategies have served as a springboard to improve cancer services beyond the COVID-19 pandemic.

\author{
Case \\ report
} Zambia

- Establishment of local taskforce

- Staff training to ensure that all staff were adequately trained in the prevention of COVID-19 transmission

- facilitation of good hygiene practices

- Adoption of a triage system

- Rethinking of patient scheduling

- Use of telehealth for meetings

Motlagh To provide recommendations and possible actions that

et al. should be considered by patients, their caregivers and

Expert consensus 
families, physician, nurses, managers and staff of medical centres involved in cancer diagnosis and treatment
Saab et al. 2020
To investigate the impact of the pandemic and its associated response on the care of children with cancer in the Middle East, North
- Patients wearing of disposable gown

- Patients wearing face masks when receiving their treatment

- Adopt a triage system

- Use teleconferencing

- Patients and their companions to wear while at the facility

- Weekly educational sessions to update staff

- Creating a call centre to answer the questions of patients and their families

- Limit the number of patient companions

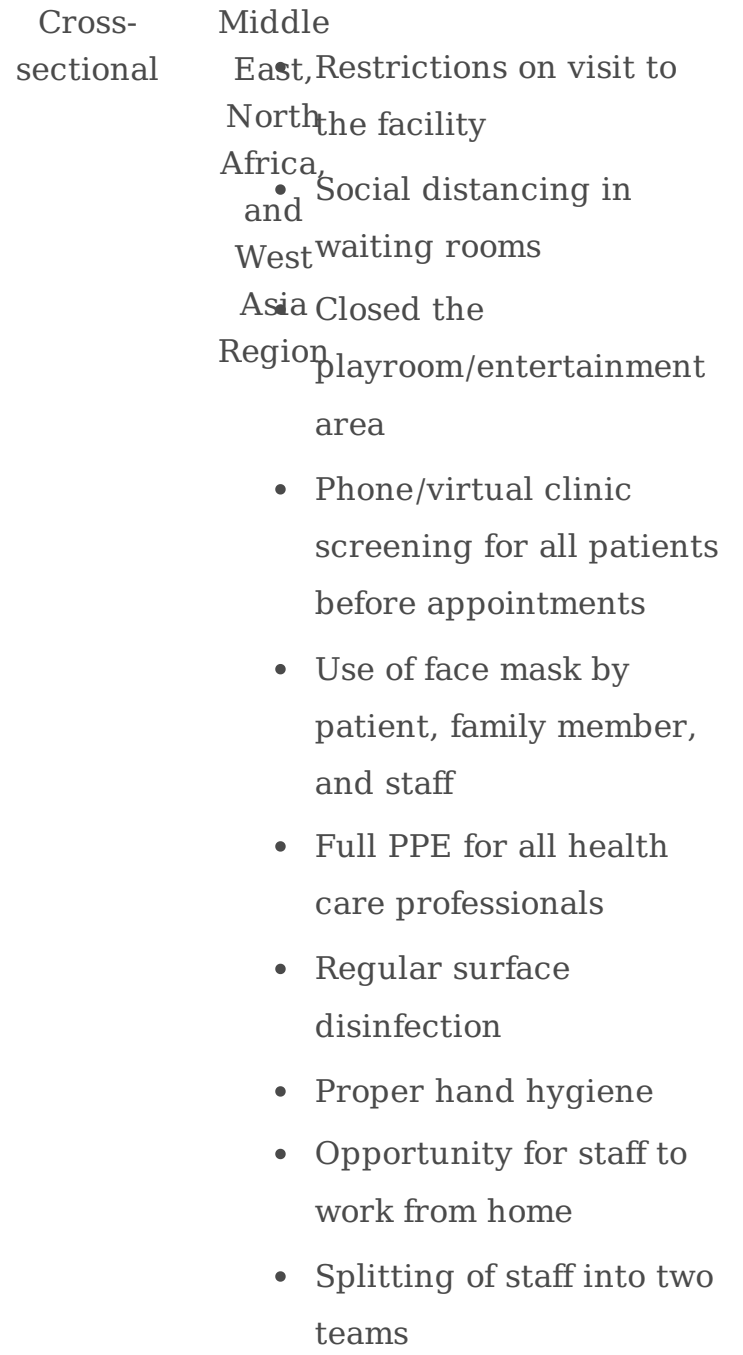


- Hypofractionation of radiotherapy when possible

- Use of telehealth for follow-up, teaching activities and multidisciplinary team meetings

Samiee et To present our policy and recommendations at a private al. 2020 radiology-oncology centre

Case
report

report

Iran

- Developed COVID-19

policies and guidelines

- Use of face mask by patient, family member, and radiotherapy staff

- Avoid, defer or shorten radiotherapy when possible

- Use of telehealth for follow-up visits

- Cancelled weekly visits for patients under treatment

- Create a direct hotline and WhatsApp discussion platform

- Adoption of a triage system

- Use of PPE

- Opportunity to work from home

- Practice physical distancing of at least 1.5 $\mathrm{m}$

- Stopped the use of physical wedges

- Dedicated clinic for further COVID-19 assessment and quarantine

Wang et To present the measures preventing and controlling

al. 2020
COVID-19 taken at our hospital over the past 2 months, as well as their corresponding effects.
Case

report
China

- Developed COVID-19 policies and guidelines 
- Training for all radiotherapy staff

- Partitioned the radiotherapy centre into zones

- Regular disinfection of the environment and equipment

- Good air ventilation

- Use of telehealth

- Adoption of a triage system

- Restriction on the number of escorts

- A cloth or disposable sheet for each patient

- Use of PPE

- COVID-19 testing

- Dedicated hospital for further COVID-19 assessment and quarantine

- Proper waste management

Wei et al. To detail our infection control experience at the 2020 radiotherapy centre of the Hubei Cancer Hospital

Case
report
China

- Formation of an ad hoc emergency infection control team

- Partitioned the clinical area of the radiotherapy centre into three zones

- Training for radiotherapy staff

- Staff rotation with no overlap

- Adoption of a triage system

- Wearing of face mask for all patients and accompanying carers

- COVID-19 testing 
- Dedicated isolation room for suspected cases

- Dedicated hospital for further COVID-19 assessment and quarantine

- Hand hygiene

- Use of telehealth for appointment, follow-up and consultations

- A strict single-patient rule

- Spacing of two meters or more at the waiting area

- Use of PPE

- Effective disinfection of the environment and equipment

- Good air ventilation

- Strict adherence of medical waste management regulations

$\begin{array}{ll}\text { Wu et al. } & \text { To briefly review the radiation therapy management in } \\ 2020 & \text { Wuhan since January 2020, with the hope that the } \\ \text { experience learned, and the lessons learned will help } \\ \text { guide practice in other regions that are or might be } \\ \text { facing outbreaks of this disease. }\end{array}$

Critical
review China

- Adoption of a triage system

- Health education fo patients

- Developed COVID-19 policies and guidelines

- Staff training

- Divided the radiotherapy centre into zones

- Single-use clear wrap for immobilisation devices

- Use of PPE
Xie et al. To report our experience and preliminary outcomes of 2020 209 RT patients, who were treated at the Zhongnan Hospital of Wuhan University (ZHWU) during the period when the city was locked down on Jan 23, 2020.

\author{
Case \\ report
} China

- Adoption of a triage system

- COVID-19 testing

- Isolation space for suspected cases 
- Dedicated hospital for further COVID-19

assessment and quarantine

- Wearing of face mask for all patients and carers

- Strict distancing of at least $1.5 \mathrm{~m}$ apart

- Daily disinfection

- A buddy system to expedite the notification of unwell team members

- Use of PPE

- Splitting of staff into teams

- Strict hand hygiene

- Effective disposal of medical hazard waste

- Prohibition of team gatherings

Table 2: Radiotherapy fractionation schedules adopted by LMICs during the COVID-19 pandemic 


\begin{tabular}{|c|c|c|c|}
\hline Authors & Country & Site & Fractionation schedules \\
\hline \multirow[t]{3}{*}{$\begin{array}{l}\text { Aghili, Jafari \& Vand } \\
\text { Rajabpoor } 2020\end{array}$} & Iran & $\begin{array}{l}\text { Head } \\
\text { and } \\
\text { Neck }\end{array}$ & $\begin{array}{l}\text { Oral tongue pT1-T2, N0: } 39 \mathrm{~Gy} / 13 \mathrm{fx} \text { a in } 7 \text { days, two times daily } \\
\text { instead of } 60 \mathrm{~Gy} / 30 \mathrm{fx} \text { by external radiotherapy. }\end{array}$ \\
\hline & & Prostate & $\begin{array}{l}\text { Monotherapy in low-risk patients: recommend delaying the } \\
\text { treatment for 3-5 months. }\end{array}$ \\
\hline & & & $\begin{array}{l}\text { High risk: Two fractions of } 13.5 \mathrm{~Gy}^{\mathrm{a}} \text { or } 15 \mathrm{GY} \text { for booster } \\
\text { dosages after external radiotherapy in one session. }\end{array}$ \\
\hline \multirow[t]{2}{*}{ Baldotto et al. 2020} & Brazil & Lung & Initial disease: $45-54 \mathrm{~Gy} / 3 \mathrm{fx}$ \\
\hline & & & $\begin{array}{l}\text { Locally advanced disease: } 60 \mathrm{~Gy} / 30 \mathrm{fx} \text { or } 55-60 \mathrm{~Gy} / 20 \text { fractions } \\
\text { or } 60 \mathrm{~Gy} / 15 \mathrm{fx} \text { (recommended) }\end{array}$ \\
\hline \multirow[t]{14}{*}{ Gupta et al. 2020} & India & $\begin{array}{l}\text { Head } \\
\text { and } \\
\text { Neck }\end{array}$ & Postoperative: $55 \mathrm{~Gy} / 25 \mathrm{fx}$ \\
\hline & & Cervix & $45 \mathrm{~Gy} / 20 \mathrm{fx}$ \\
\hline & & & Two sessions of 9 Gy each delivered one week apart ${ }^{a}$ \\
\hline & & Brain & High grade: $40 \mathrm{~Gy} / 15 \mathrm{fx}$ \\
\hline & & Rectum & Preoperative, short course: 25 Gy/5 fx (recommended) \\
\hline & & & Postoperative: $45 \mathrm{~Gy} / 20 \mathrm{fx}$ \\
\hline & & Breast & Whole breast only: $26 \mathrm{~Gy} / 5 \mathrm{fx}$ \\
\hline & & & Chest wall only: $26 \mathrm{~Gy} / 5 \mathrm{fx}$ \\
\hline & & & Nodal irradiation needed: $40 \mathrm{~Gy} / 15 \mathrm{fx}$ \\
\hline & & Palliative & Painful bone metastasis: $8 \mathrm{~Gy} / 1 \mathrm{fx}$ \\
\hline & & & Spinal cord compression $<48 \mathrm{hr}: 8 \mathrm{~Gy} / 1 \mathrm{fx}$ \\
\hline & & & Symptomatic brain metastasis: $8 \mathrm{~Gy} / 1 \mathrm{fx}$ \\
\hline & & & Tumour bleed: $8 \mathrm{~Gy} / 1 \mathrm{fx}$ \\
\hline & & & $\begin{array}{l}\text { Superior vena cava obstruction (symptomatic patients only): } 20 \\
\text { Gy/5 fx }\end{array}$ \\
\hline \multirow[t]{6}{*}{ Lombe et al 2020} & Zambia & Breast & Chest wall: $50 \mathrm{~Gy} / 25 \mathrm{fx}$ or $28.5 \mathrm{~Gy} / 5 \mathrm{fx}$ (recommended) \\
\hline & & & $\begin{array}{l}\text { Supraclavicular + chest wall: } 50 \mathrm{~Gy} / 25 \mathrm{fx} \text { or } 40 \mathrm{~Gy} / 10 \mathrm{fx} \\
\text { (recommended) }\end{array}$ \\
\hline & & Cervix & 50 Gy/25 fx or 41.25 Gy/15 fx (recommended) \\
\hline & & & $\begin{array}{l}7 \text { Gy/4 fx or } 8 \text { Gy x } 3 \text {; } 9 \text { Gy x } 2 \text { one week apart; } 9.4 \text { Gy x } 2 \text { one } \\
\text { week apart (recommended) a }\end{array}$ \\
\hline & & Prostate & High risk: $74 \mathrm{~Gy} / 37 \mathrm{fx}$ or $60 \mathrm{~Gy} / 20 \mathrm{fx}$ (recommended) \\
\hline & & Palliative & $\begin{array}{l}\text { Spinal cord compression: } 20 \mathrm{~Gy} / 5 \mathrm{fx} \text { or } 30 \mathrm{~Gy} / 5 \mathrm{fx} \text { or } 8 \mathrm{~Gy} / 1 \mathrm{fx} \\
\text { (recommended) }\end{array}$ \\
\hline
\end{tabular}

a: Dose of brachytherapy; Fx: Fraction; Gy: Gray

\section{Appendix}




\section{Figures}

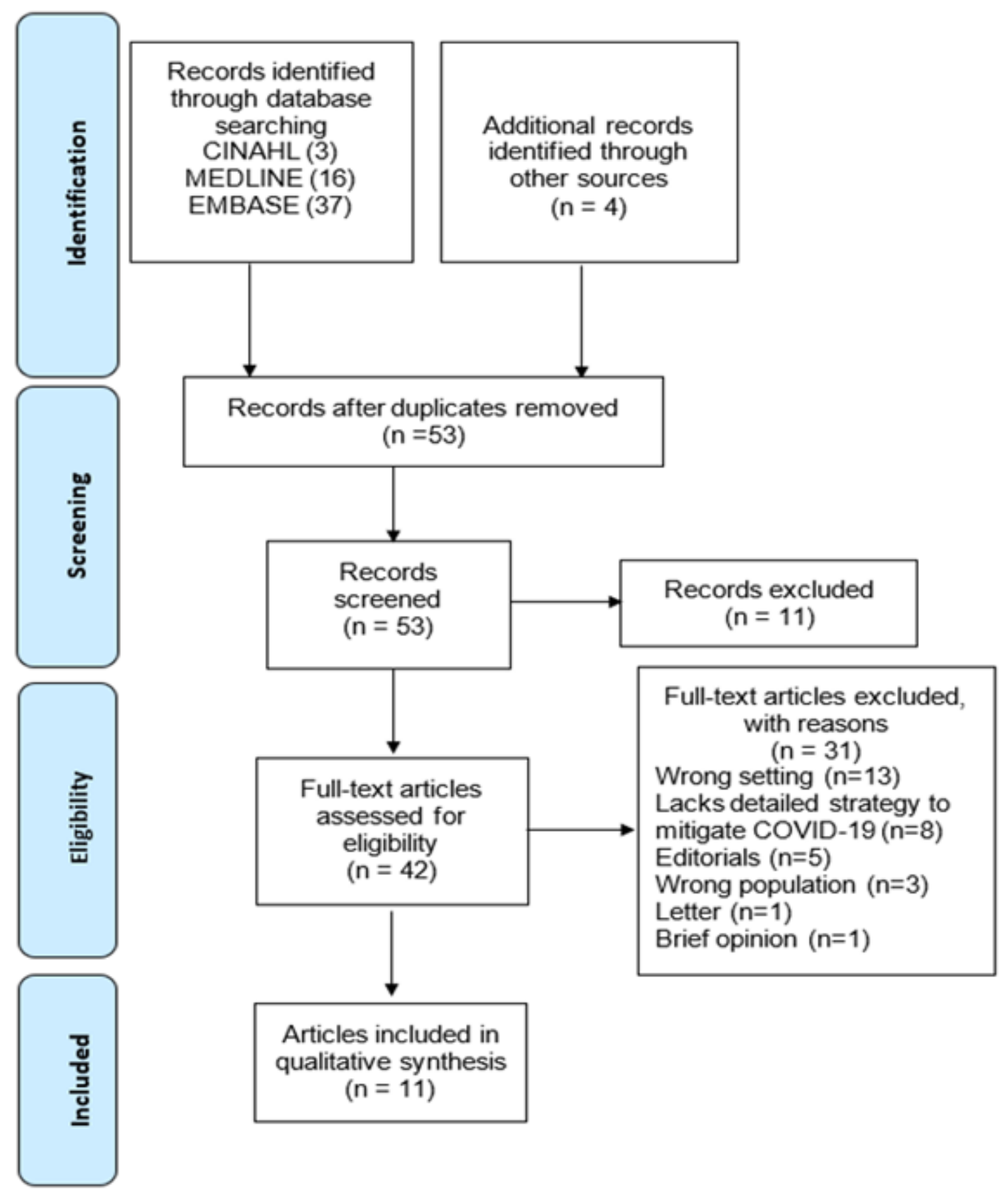

Figure 1

PRISMA flow diagram 


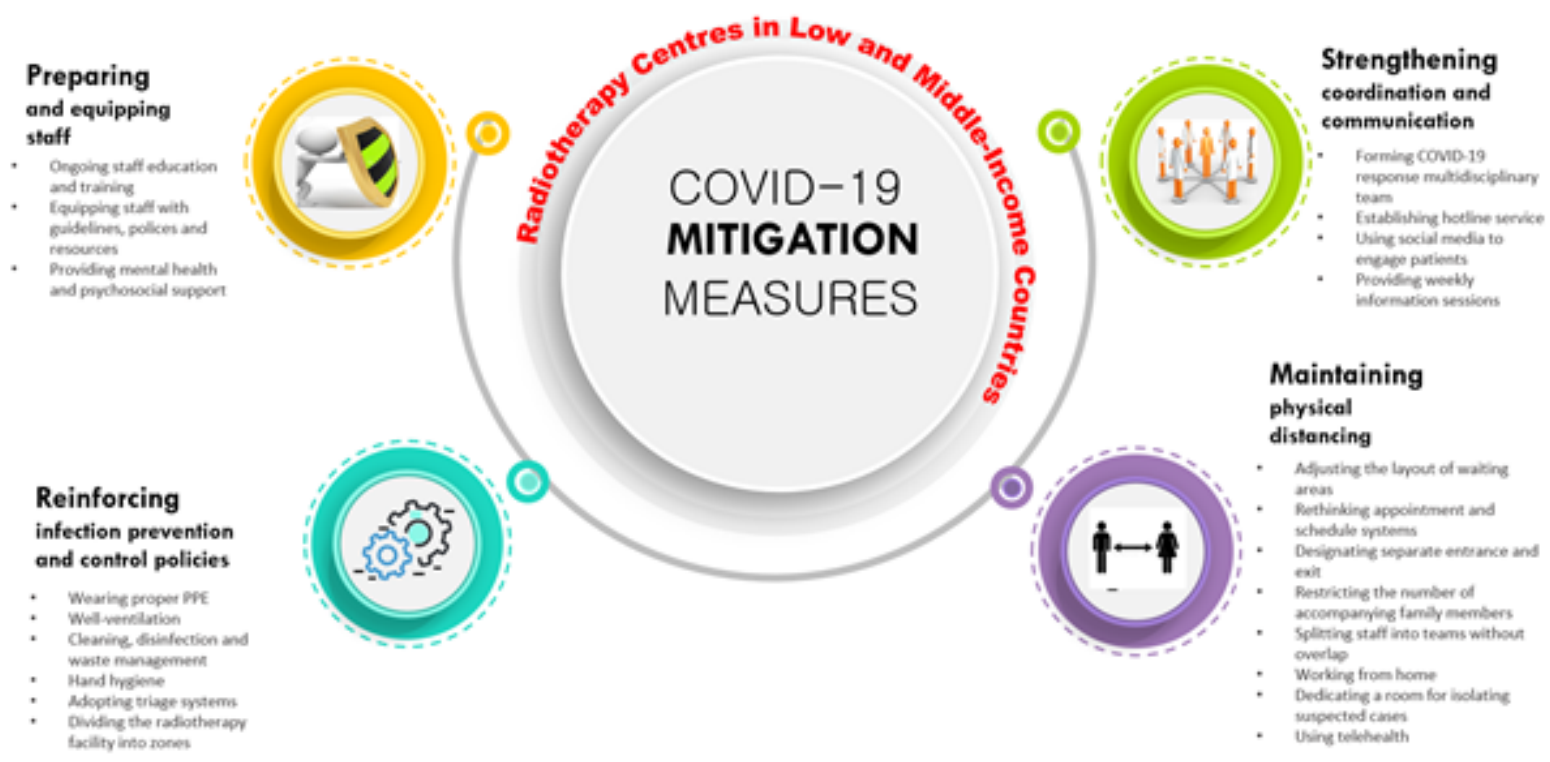

Figure 2

COVID-19 mitigation measures adopted by radiotherapy centres in LMICs 